\title{
Extension urbaine et densité de la population à Kinshasa : contribution de la télédétection \\ satellitaire
}

Urban sprawl and population density in Kinshasa: the contribution of satellite teledetection

\section{Eléonore Wolff et Virginie Delbart}

\section{(2) OpenEdition \\ Journals}

\section{Édition électronique}

URL : https://journals.openedition.org/belgeo/15451

DOI : 10.4000/belgeo.15451

ISSN : 2294-9135

\section{Éditeur :}

National Committee of Geography of Belgium, Société Royale Belge de Géographie

Édition imprimée

Date de publication : 30 mars 2002

Pagination : 45-99

ISSN : 1377-2368

Référence électronique

Eléonore Wolff et Virginie Delbart, «Extension urbaine et densité de la population à Kinshasa :

contribution de la télédétection satellitaire », Belgeo [En ligne], 1 | 2002, mis en ligne le 31 mars 2002, consulté le 14 juin 2021. URL : http://journals.openedition.org/belgeo/15451 ; DOI : https://doi.org/ $10.4000 /$ belgeo. 15451

Ce document a été généré automatiquement le 14 juin 2021.

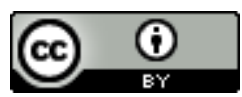

Belgeo est mis à disposition selon les termes de la licence Creative Commons Attribution 4.0 International. 


\title{
Extension urbaine et densité de la population à Kinshasa : contribution de la télédétection satellitaire
}

\author{
Urban sprawl and population density in Kinshasa: the contribution of satellite \\ teledetection
}

Eléonore Wolff et Virginie Delbart

Les auteurs remercient vivement Eric Lambin, responsable de l'équipe "Remote Sensing and Land use changes » en géographie à l'Université catholique de Louvain, pour avoir mis à la disposition de Virginie Delbart les images SPOT XS et P (KJ 096-359) du 31 mars 1995. Elles remercient également très sincèrement les chercheurs kinois qui, malgré leurs conditions de vie difficiles, ont accepté d'aider Virginie Delbart lors de son travail de terrain, ainsi que Marie Sintzoff qui a appuyé les traitements d'images et la réalisation des cartes.

1 La situation démographique de la capitale de la République Démocratique du Congo est semblable à celle prévalant dans beaucoup de villes d'Afrique tropicale ; la population continue de croître rapidement depuis l'indépendance (Vennetier, 1991). La crise socioéconomique y engendre des conditions de vie très difficiles : développement du secteur informel, déstructuration des réseaux de transport en commun et extrême croissance spatiale de la ville entraînant des trajets de plus en plus longs vers le centre-ville, lieu de concentration de toutes les activités administratives, commerciales...

2 Il y a cependant une différence entre Kinshasa et la majorité des autres capitales africaines. Ces dernières années, la tendance démographique serait à la diminution de la croissance de la population (Bruneau, 1995). Cet avis ne rassemble pas néanmoins l'unanimité chez les scientifiques (de Saint Moulin et al., 1995), mais en l'absence de données de population plus récentes que celles issues du recensement scientifique de la population de 1984, il est difficile de trancher. Néanmoins, on parle de contreurbanisation, c'est-à-dire d'une réorientation des personnes depuis les grandes villes vers les villes moyennes et petites, voire vers le milieu rural (Dubresson et Raison, 1998). Quoi qu'il en soit, en République Démocratique du Congo, on peut affirmer que 
les villes secondaires ne sont que de faibles contre-poids à la croissance de la capitale ; en effet, elles possèdent peu d'infrastructures de base et ne parviennent pas à contrebalancer l'effet polarisant de Kinshasa (Wolff et al., 2001).

3 A Kinshasa, le taux de croissance annuel moyen était d'environ $8 \%$ entre 1960 et 1980 (Mwanza Wa Mwanza, 1996); il est estimé à 4 à $5 \%$ depuis les années 80 (Nzuzi Lelo, 1991 ; Luzolele et al., 1999). De tels taux de croissance auraient engendré une population d'environ six millions d'habitants en 2000, ce qui élève Kinshasa à la seconde place, donc après Lagos, parmi les villes les plus peuplées d'Afrique noire. Nous parlons d'estimations car il n'y a pas de données fiables plus récentes que celles du recensement scientifique de la population de 1984. Cette situation est une moyenne entre les données officielles de l'Hôtel de Ville de Kinshasa et les projections (Ngondo et al., 1992 ; INS, 1993).

4 Une telle croissance démographique a des conséquences sur la morphologie de l'espace kinois. Deux processus s'observent :

- la croissance de la population engendre une extension spatiale de la ville ;

- la croissance de la population, dans un contexte de rareté des opportunités d'emploi, d'insécurité, de concentration des infrastructures et de difficultés croissantes de transport conduit à la densification des quartiers existants.

5 L'objectif de cet article est de tenter d'établir l'importance relative de l'extension urbaine et de la densification. C'est pourquoi, les cartes existantes et une image satellitaire récente à haute résolution ont été utilisées pour observer la croissance spatiale de la surface urbanisée. Les statistiques de population disponibles seront utilisées pour calculer la densité de la population. Une mission de terrain a été effectuée à Kinshasa en février 2000 afin d'analyser les conséquences de la croissance démographique dans les communes centrales et périphériques de la ville, mais aussi afin de recueillir des données officielles, d'enquêter dans différentes communes de Kinshasa, de rassembler des documents et connaissances de différents chercheurs travaillant activement dans un secteur ou l'autre en rapport avec les conséquences de la croissance démographique sur l'espace kinois.

\section{Cartes et images}

6 L'extension spatiale de la ville est mal connue d'un point de vue cartographique. La carte «Ville de Kinshasa - Zones de santé » (carte au 1:20 000 éditée en 1969 et révisée en 1997 d'après le fond de base du Plan de la Ville de Kinshasa, édition provisoire, publiée par l'Institut Géographique du Zaïre) porte le tracé des limites communales de la zone urbanisée en 1969. L'atlas de Kinshasa (Flouriot et al., 1975) permet de comprendre l'organisation urbaine des années 70. Ces deux documents fournissent des informations intéressantes, mais complètement dépassées par la situation actuelle.

7 L'utilisation d'images satellitaires constitue l'alternative idéale en l'absence de documents cartographiques récents ou assez détaillés. Les données satellitaires utilisées lors de cette étude sont deux images Spot (KJ 096-359), datant du 31 mars 1995, enregistrées simultanément en mode panchromatique et multi-spectral. Afin d'être interprétées, ces images ont été prétraitées. 


\section{Correction géométrique et rehaussement de contrastes}

8 Le géocodage est destiné à corriger l'inclinaison de quelques degrés de l'orbite du satellite par rapport au Nord géographique et à attribuer des coordonnées cartographiques à l'image. Pour réaliser ce géocodage, nous avons repéré 50 points de calage sur la carte «Ville de Kinshasa - Zones de santé » et sur l'image panchromatique. Ces points doivent être aisément repérables, tant sur la carte que sur l'image, et ne pas avoir changé entre la conception de la carte et l'enregistrement de l'image. Ces points sont forcément localisés sur la zone couverte par la carte, zone beaucoup plus restreinte que celle couverte par l'image. De plus, les zones périphériques de 1969 se sont considérablement densifiées; il devient impossible d'y repérer des cours d'eau ou des routes utilisés comme points de calage. En conséquence, les points de calage sont concentrés sur la partie centrale de la zone couverte par la carte, soit sur l'extension de la ville en 1969.

L'image SPOT Panchromatique a été corrigée selon un modèle polynomial d'ordre 1 par la méthode du plus proche voisin. L'erreur quadratique moyenne est de 1.54 pixels en $\mathrm{X}$ et $1.49 \mathrm{en} \mathrm{Y}$. Cette erreur est probablement plus importante en périphérie qu'au centreville (où ont été repérés les points de calage). Cependant, en l'absence de données cartographiques précises de référence, il est difficile de quantifier l'erreur géométrique en périphérie.

Ensuite, l'image SPOT multi-spectrale a été corrigée en utilisant l'image panchromatique corrigée comme référence. 30 points de calage ont été repérés sur l'ensemble de l'image. L'image a été corrigée selon un modèle polynomial d'ordre 2 par la méthode du plus proche voisin. L'erreur quadratique moyenne est de 0.31 pixels en $\mathrm{X}$ et de 0.49 en Y.

11 Les contrastes ont été améliorés de façon globale sur l'ensemble de l'image, ce qui est satisfaisant pour délimiter la tache urbaine. Pour visualiser les contrastes au sein des zones densément bâties, un rehaussement particulier a été effectué sur le centre-ville.

\section{Fusion des images panchromatique et multi-spectral}

La fusion des deux modes trouve son intérêt dans la combinaison de leurs avantages respectifs. L'image panchromatique n'a qu'une seule bande spectrale enregistrée dans le spectre visible, mais sa résolution spatiale est de 10 mètres ; la texture et la structure des éléments sont bien mises en évidence. L'image multi-spectrale n'a qu'une résolution spatiale de 20 mètres, mais a trois bandes spectrales dans les longueurs d'onde $\mathrm{du}$ vert, rouge et infrarouge; elle apporte l'information nécessaire pour déterminer les types d'occupation du sol. Ces deux images ont été fusionnées par la méthode IHS-RGB afin d'optimiser les conditions de l'interprétation visuelle.

\section{Tracé des limites communales}


Figure 1. Tracé des limites communales en 1969.

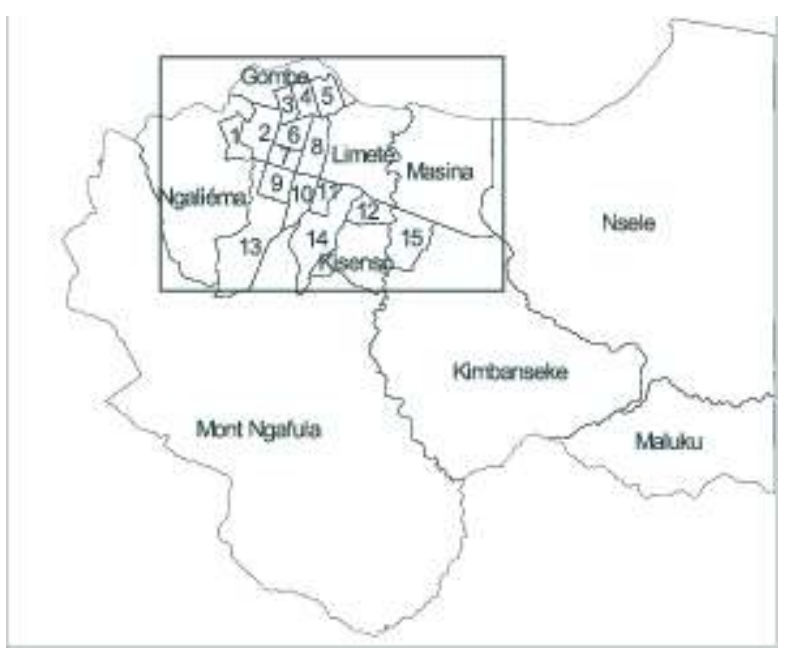

D'après la carte au 1 : 20000 éditée en 1969 et révisée en 1997 d'après le fond de base du Plan de la Ville de Kinshasa, édition provisoire, publiée par l'Institut Géographique du Zaïre ; et en 1995, extension de la carte de 1969 et extension de la zone d'étude.

Les limites des communes centrales ont été reportées sur l'image fusionnée par interprétation visuelle en se basant sur la carte " Ville de Kinshasa - Zones de santé » et sur la description de leur tracé par l'arrêté ministériel (arrêté ministériel n $69-0042$ Moniteur Congolais du 23/01/1969). Les limites des autres communes, ne figurant pas sur la carte de 1969, ont été tracées en utilisant les documents suivants :

- arrêté ministériel $n^{\circ}$ 68-430 publié au Moniteur Congolais du 02-12-1968 fixant la délimitation officielle de la Ville de Kinshasa,

- arrêté ministériel n 69-0042 publié au Moniteur Congolais du 23/01/1969 fixant la délimitation officielle des 24 communes de la Ville de Kinshasa,

- carte du territoire de Kasangulu établie au 1:200 000, sur base de photographies aériennes prises en 1950 et 1953 par l'Institut Géographique du Zaïre où l'on peut repérer le tracé complet de la Ville de Kinshasa.

14 C'est ainsi que le contour de six communes périphériques, incomplet sur la carte de 1969, a été tracé ; il s'agit des communes de Kimbanseke, Kisenso, Lemba, Mont Ngafula, Selembao et Ngaliéma. Pour les deux grandes communes rurales de Nsele et Maluku dépassant de la zone couverte par l'image satellitaire, le tracé de leurs limites n'a pu être clôturé.

Sur base de ce tracé, les superficies communales ont été calculées et comparées à celles figurant dans le recensement de 1984 (INS, 1992). Dans la majorité des cas, les différences sont négligeables (tableau 1); elles peuvent être attribuées à de petites erreurs lors de notre digitalisation des limites communales ou lors de la détermination des superficies par les autorités administratives. Pour la commune de la Gombe, dont la superficie passe de $192,2 \mathrm{~km}^{2}$ (INS, 1992) à $12,3 \mathrm{~km}^{2}$, il s'agit très probablement d'une erreur d'encodage dans les statistiques de l'INS. 
Tableau 1. Superficies des 24 communes telles que définies dans le recensement de 1984 par l'INS comparées aux résultats de la digitalisation sur l'image satellitaire SPOT.

\begin{tabular}{lccc} 
Communes & $\begin{array}{c}\text { Superficie en } \mathrm{km}^{2} \\
\text { - I.N.S. }\end{array}$ & $\begin{array}{c}\text { Superficie en } \mathrm{km}^{2} \\
\text { sur image }\end{array}$ & $\begin{array}{c}\text { Rapport } \\
\text { S INS/S image }\end{array}$ \\
\hline Gombe & 192,2 & 12,3 & 15,6 \\
Lemba & 23,7 & 14,1 & 1,7 \\
Masina & 69,7 & 47,4 & 1,5 \\
Ngaliéma & 81,7 & 62,6 & 1,3 \\
Selembao & 23,2 & 19,5 & 1,2 \\
Kasa-Vubu & 5,0 & 4,0 & 1,2 \\
Ndjili & 11,4 & 9,4 & 1,2 \\
Barumbu & 4,7 & 4,3 & 1,1 \\
Kalamu & 6,6 & 6,0 & 1,1 \\
Ngiri-Ngiri & 3,4 & 2,9 & 1,1 \\
Bumbu & 5,3 & 4,6 & 1,1 \\
Makala & 5,6 & 5,1 & 1,1 \\
Ngaba & 3,2 & 2,9 & 1,1 \\
Kintambo & 3,9 & 4,0 & 1,0 \\
Lingwala & 2,9 & 2,8 & 1,0 \\
Bandalungwa & 6,8 & 7,0 & 1,0 \\
Limete & 27,1 & 28,3 & 1,0 \\
Matete & 4,9 & 4,8 & 1,0 \\
Kisenso & 16,6 & 16,1 & 1,0 \\
Kinshasa & 2,9 & 3,2 & 0,9 \\
Mont Ngafula & 358,9 & 424,4 & 0,9 \\
Kimbanseke & 76,9 & 181,7 & 0,4 \\
& & &
\end{tabular}

16 Pour les communes longeant le fleuve, les différences observées pourraient être causées par la prise en compte par l'INS des parties du fleuve entrant dans la délimitation légale des communes, tandis que ces parties sont exclues de notre digitalisation (aucune indication ne nous permettant de digitaliser la limite de la partie fluviale des communes). Cependant, la prise en compte de la partie fluviale ne devrait pas justifier de telles différences et devrait conduire systématiquement à une surestimation des superficies communales dans les données de l'INS. Pour la commune de Ngaliéma, il semble plus vraisemblable d'imaginer qu'il y a une erreur dans le positionnement de la décimale; une superficie de $19,2 \mathrm{~km}^{2}$ dans les données INS conduirait à surestimation avec un rapport de 1,6 en comparaison avec la superficie sur l'image. Cette surestimation est proche de celles des communes de Lemba et de Masina ; elle reste néanmoins forte. Finalement, les superficies des communes de Mont Ngafula et de Kimbanseke sont surestimées par rapport à celle de l'INS et constituent un contreexemple; ce sont deux communes beaucoup plus grandes dont une grande partie est encore rurale.

17 Pour les communes ne longeant pas le fleuve, nos entretiens sur le terrain, ainsi que la lecture de l'introduction du recensement de l'INS, laissent imaginer un niveau de précision relativement faible des mesures faites par l'administration.

\section{Extension de Kinshasa}

18 Une classification non supervisée de l'image SPOT multi-spectrale de 1995 en 20 classes a permis de distinguer la tache urbaine de Kinshasa en discriminant le bâti du non-bâti (fig. 2) ; une des classes spectrales a été divisée à l'aide d'un masque afin de distinguer 
des zones bâties à l'Ouest de zones défrichées à l'Est. Afin de généraliser un peu les résultats, un filtre modal préservant les alignements a été appliqué sur l'image du bâti dans une fenêtre de $3 \times 3$ pixels et les polygones inférieurs à 1 ha ont été fusionnés avec les polygones adjacents.

Figure 2. Classification du bâti, extension de la ville en 1969 et limites communales.
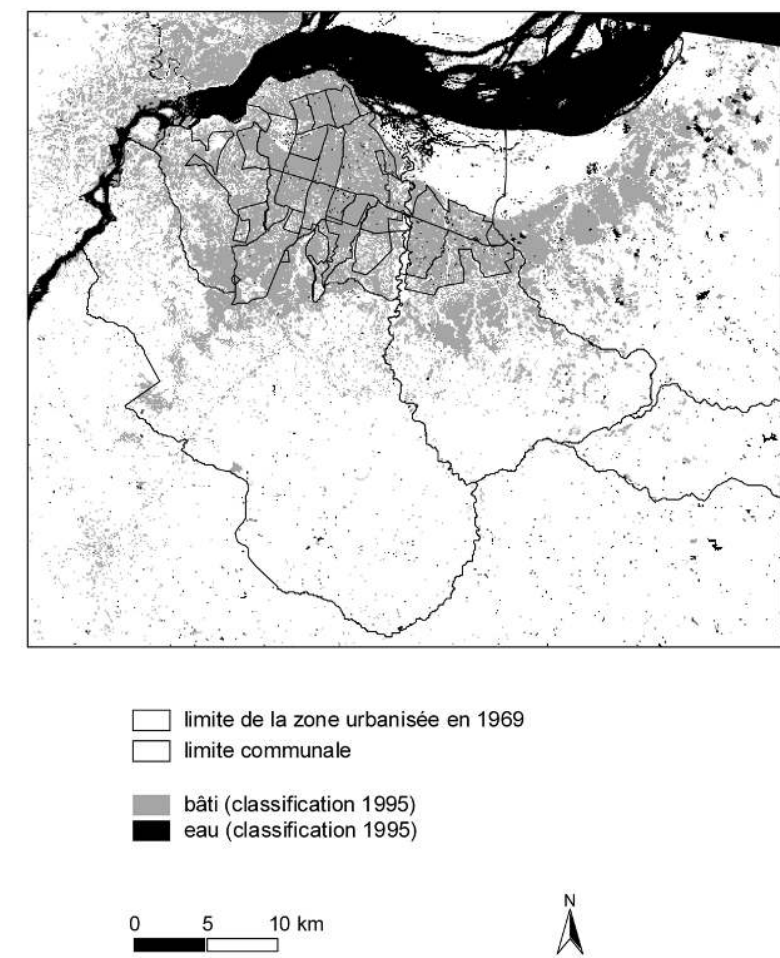

Etant donné les difficultés logistiques considérables, il était impossible de valider ce résultat durant la mission de terrain sur toute l'agglomération de Kinshasa. Cependant, la mission de terrain et l'analyse des images SPOT ont permis d'acquérir une bonne connaissance de la ville et de son extension, ainsi qu'une bonne capacité d'interprétation visuelle des différents quartiers de la ville; cette méthode permet de cerner qualitativement les limites de la classification du bâti.

La surface bâtie dans la zone collinaire des communes du Sud et de l'Ouest est sousestimée. Pour retenir le sol sablonneux qui a tendance à s'éroder lors des pluies abondantes (glissement de terrains entraînant habitations et infrastructures), les habitants plantent une végétation arborée, composée d'arbres fruitiers (manguiers, avocatiers, palmiers...) et de bambous de Chine ; cette végétation y perturbe la réponse spectrale du «bâti ».

La surface bâtie est surestimée dans les communes de l'Est, essentiellement pour les quartiers Mpasa dans la commune de Nsele. Pour limiter l'extension de la ville vers l'Est, l'administration kinoise avait prévu des aménagements de base (eau, électricité, ...) dans cette zone. Les Kinois y ont acheté des parcelles, qu'ils ont défrichées dans la perspective d'y construire des habitations lorsque les aménagements prévus auraient été effectués. Cependant, la crise a laissé ces aménagements en suspens depuis plus de dix ans. Les parcelles défrichées se caractérisent par une signature spectrale trop 
proche de celle des zones urbanisées pour pouvoir les distinguer, d'où une certaine surestimation de la surface bâtie. bâties (12-26 \%) ; en réalité, le bâti est réparti de façon très inégale. Les deux tiers de la commune de Masina en bordure du fleuve sont occupés par des marécages, le reste est très densément bâti. La commune de Kimbanseke est densément bâtie à proximité du Boulevard Lumumba, mais plus on s'en écarte plus la densité du bâti diminue, le Sud de la commune étant quasi inhabité. La commune de Mont Ngafula est la moins bâtie (12\%); l'habitat y est dispersé dans les collines à l'exception des zones proches des voies de communication ou du centre-ville qui sont densément bâties. 
Tableau 2. Superficie communale, superficie bâtie, pourcentage bâti dans la commune et typologie des communes de Flouriot (1975).

\begin{tabular}{lcccl} 
Communes & $\begin{array}{c}\text { Superficie } \\
\text { communale } \\
\text { en } \mathrm{km}^{2}\end{array}$ & $\begin{array}{c}\text { Superficie bâtie } \\
\text { par commune } \\
\text { en km² }\end{array}$ & $\begin{array}{c}\text { \% bâti } \\
\text { par commune }\end{array}$ & $\begin{array}{c}\text { Typologie des communes } \\
\text { (Flouriot, 1975) }\end{array}$ \\
\hline Ngiri-Ngiri & 3,0 & 2,9 & 97 & Nouvelles cités (30-50) \\
Ngaba & 2,9 & 2,8 & 95 & Extension Sud-Ouest \\
Kinshasa & 3,2 & 3,1 & 95 & Anciennes cités (10-30) \\
Kalamu & 6,0 & 5,6 & 95 & Cités planifiées (50-60) \\
Barumbu & 4,3 & 4,0 & 93 & Anciennes cités (10-30) \\
Bumbu & 4,6 & 4,2 & 91 & Extension Sud-Ouest \\
Kasa-Vubu & 4,0 & 3,6 & 90 & Nouvelles cités (30-50) \\
Kintambo & 4,0 & 3,4 & 87 & Anciennes cités (10-30) \\
Lingwala & 2,8 & 2,4 & 87 & Anciennes cités (10-30) \\
Lemba & 14,1 & 11,9 & 85 & Cités planifiées (50-60) \\
Makala & 5,1 & 4,3 & 84 & Extension Sud-Ouest \\
Matete & 4,8 & 3,9 & 83 & Cités planifiées (50-60) \\
Selembao & 19,5 & 15,5 & 79 & Extension Sud-Ouest \\
Gombe & 12,3 & 9,4 & 77 & Résidentiel Haut standing \\
Ndjili & 9,4 & 6,9 & 74 & Cités planifiées (50-60) \\
Bandalungwa & 7,0 & 5,1 & 72 & Cités planifiées (50-60) \\
Ngaliéma & 62,6 & 41,4 & 66 & Résidentiel Haut standing \\
Limete & 28,3 & 18,3 & 65 & Résidentiel Haut standing \\
Kisenso & 16,1 & 9,1 & 57 & Extension Est \\
Masina & 47,4 & 12,2 & 26 & Extension Est \\
Kimbanseke & 181,7 & 46,0 & 25 & Extension Est \\
Mont Ngafula & 424,4 & 49,2 & 12 & Extension Sud-Ouest \\
& & & &
\end{tabular}

\section{Densité de population}

La répartition du bâti dans les communes rurales étant fort inégale, il est intéressant de calculer la densité de population par rapport à l'espace bâti en 1995 (tableau 3). 
Tableau 3. Densité de la population en 1995 calculée par rapport à la superficie communale et par rapport à la superficie bâtie.

$\begin{array}{ccc}\text { Communes Population projetée } & \begin{array}{c}\text { Densité de population } \\ \text { (hab. } / \mathrm{km}^{2} \text { commune) }\end{array} & \begin{array}{c}\text { Densité de population } \\ \text { (hab./ } / \mathrm{km}^{2} \text { bâti) }\end{array}\end{array}$

\begin{tabular}{|c|c|c|c|}
\hline Ngiri-Ngiri & 145801 & 49424 & 50979 \\
\hline Kalamu & 284716 & 47771 & 50481 \\
\hline Bumbu & 201896 & 43986 & 48301 \\
\hline Ngaba & 131884 & 45166 & 47441 \\
\hline Matete & 185836 & 38959 & 47166 \\
\hline Makala & 192987 & 37546 & 44570 \\
\hline Kinshasa & 132346 & 40974 & 43250 \\
\hline Ndjili & 278146 & 29716 & 40194 \\
\hline Kasa-Vubu & 132665 & 33249 & 36954 \\
\hline Lingwala & 87111 & 31111 & 35848 \\
\hline Bandalungwa & 172216 & 24637 & 34102 \\
\hline Barumbu & 122495 & 28554 & 30624 \\
\hline Kintambo & 87330 & 22053 & 25387 \\
\hline Lemba & 283044 & 20131 & 23706 \\
\hline Kisenso & 208638 & 12975 & 22952 \\
\hline Masina & 280041 & 5909 & 22898 \\
\hline Selembao & 224254 & 11488 & 14468 \\
\hline Kimbanseke & 625715 & 3444 & 13615 \\
\hline Limete & 227103 & 8014 & 12404 \\
\hline Ngaliéma & 446689 & 7131 & 10787 \\
\hline Gombe & 30754 & 2502 & 3261 \\
\hline Mont Ngafula & 93571 & 220 & 1900 \\
\hline Maluku (*) & 93316 & I & I \\
\hline Nsele $(*)$ & 51308 & I & I \\
\hline
\end{tabular}

Pour ce faire, les effectifs de population de 1995 par commune, issus des projections réalisées par l'INS (1993), ont été divisés par la superficie bâtie de chaque commune. Cette densité a l'avantage de ne pas tenir compte des grandes zones rurales des communes périphériques, ni de la localisation des grandes surfaces inhabitées, ni des superficies occupées par le fleuve; elle est donc beaucoup plus proche des densités urbaines vécues par la population. Par exemple, pour la commune de Kimbanseke, la densité de population n'est que de $3444 \mathrm{hab} . / \mathrm{km}^{2}$ si on prend en compte toute la superficie communale, mais s'élève à $13615 \mathrm{hab} . / \mathrm{km}^{2}$ quand on ne tient compte que de la superficie bâtie; ce qui illustre beaucoup mieux la réalité des conditions de vie des habitants de cette commune. En guise de comparaison, les densités ont également été recalculées par rapport aux superficies communales calculées d'après notre tracé (cf. tableau 1). La figure 3a montre la densité de population par rapport à la superficie communale en 1995 alors que la figure 3b illustre la densité de la population calculée par rapport à la superficie bâtie de la commune en 1995. 
Figures 3 a et b. Densité de la population en 1995 calculée par rapport à la superficie communale et par rapport à la superficie bâtie.

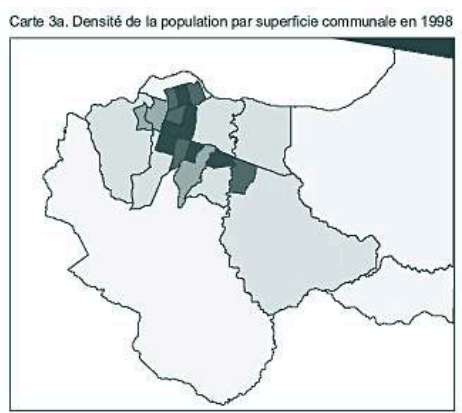

Carte 3b. Densité de la population par superficie communale bâtie en 1998

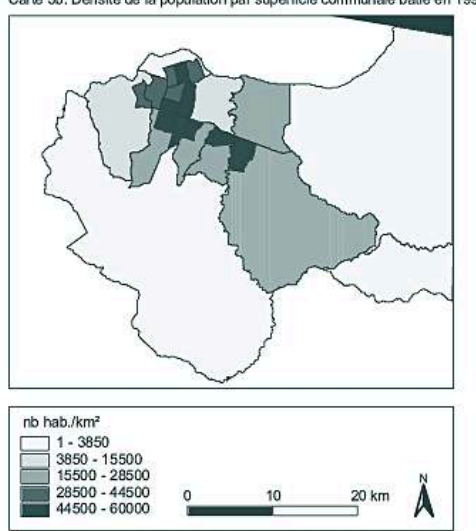

30 Sans tenir compte de la répartition du bâti, on peut, au départ des données de population existantes pour 1970, 1984 et des projections pour 1995, tenter de recalculer les densités de population grâce aux superficies communales corrigées et essayer de se prononcer sur une hypothèse de densification de certaines communes (tableau 4). 
Tableau 4. Superficies communales, effectif de population et densité de population pour 1970, 1984 et 1995 (sources : de Saint Moulin, Léon ; 1976, I.N.S. ; 1992, I.N.S., 1993).

\begin{tabular}{|c|c|c|c|c|c|c|c|}
\hline Communes & Superficie & $\begin{array}{l}1970 \\
\text { (hab.) }\end{array}$ & $\begin{array}{l}1984 \\
\text { (hab.) }\end{array}$ & $\begin{array}{c}\text { Population } \\
\text { projetée } \\
1995 \\
\text { (hab.) }\end{array}$ & $\begin{array}{c}\text { Densité } \\
\text { de pop. } \\
1970 \\
\left(\mathrm{hab} / \mathrm{km}^{2}\right)\end{array}$ & $\begin{array}{c}\text { Densité } \\
\text { de pop. } \\
1984 \\
\left(\mathrm{hab} / \mathrm{km}^{2} \text { ) }\right.\end{array}$ & $\begin{array}{c}\text { Densité } \\
\text { de pop. } \\
1995 \\
\left(\mathrm{hab} / \mathrm{km}^{2}\right)\end{array}$ \\
\hline Ngiri-Ngiri & 2,95 & 64272 & 82303 & 145801 & 21787 & 27899 & 49424 \\
\hline Kalamu & 5,96 & 100441 & 160719 & 284716 & 16853 & 26966 & 47771 \\
\hline Ngaba & 2,92 & 36702 & 74447 & 131884 & 12569 & 25496 & 45166 \\
\hline Bumbu & 4,59 & 61366 & 113968 & 201896 & 13369 & 24830 & 43986 \\
\hline Kinshasa & 3,23 & 73826 & 74708 & 132346 & 22856 & 23129 & 40974 \\
\hline Matete & 4,77 & 63369 & 104902 & 185836 & 13285 & 21992 & 38959 \\
\hline Makala & 5,14 & 49346 & 108939 & 192987 & 9600 & 21194 & 37546 \\
\hline Kasa-Vubu & 3,99 & 67525 & 74888 & 132665 & 16924 & 18769 & 33249 \\
\hline Lingwala & 2,8 & 46209 & 49173 & 87111 & 16503 & 17562 & 31111 \\
\hline Ndjili & 9,36 & 102881 & 157010 & 278146 & 10992 & 16775 & 29716 \\
\hline Barumbu & 4,29 & 59553 & 69147 & 122495 & 13882 & 16118 & 28554 \\
\hline Bandalungwa & 6,99 & 60243 & 97214 & 172216 & 8618 & 13908 & 24637 \\
\hline Kintambo & 3,96 & 38748 & 49297 & 87330 & 9785 & 12449 & 22053 \\
\hline Lemba & 14,06 & 61607 & 159775 & 283044 & 4382 & 11364 & 20131 \\
\hline Kisenso & 16,08 & 39578 & 117774 & 208638 & 2461 & 7324 & 12975 \\
\hline Selembao & 19,52 & 46908 & 126589 & 224254 & 2403 & 6485 & 11488 \\
\hline Limete & 28,34 & 41340 & 128197 & 227103 & 1459 & 4524 & 8014 \\
\hline Ngaliéma & 62,64 & 63844 & 252151 & 446689 & 1019 & 4025 & 7131 \\
\hline Masina & 47,39 & 36158 & 158080 & 280041 & 763 & 3336 & 5909 \\
\hline Kimbanseke & 181,66 & 83006 & 353209 & 625715 & 457 & 1944 & 3444 \\
\hline Gombe & 12,29 & 22615 & 17360 & 30754 & 1840 & 1413 & 2502 \\
\hline Mont Ngafula & 424,41 & 29811 & 52820 & 93571 & 70 & 124 & 220 \\
\hline Maluku (*) & 1 & 14678 & 52676 & 93316 & 1 & 1 & 1 \\
\hline Nsele $(*)$ & 1 & 24096 & 28963 & 51308 & 1 & I & I \\
\hline
\end{tabular}

(*) communes non couvertes entièrement par l'image satellitaire utilisée

31 La densité de toutes les communes de Kinshasa augmente, mais elle atteint des valeurs supérieures à 20000 hab. $/ \mathrm{km}^{2}$ en 1995 pour les petites communes déjà largement bâties en 1969, c'est-à-dire les cités et extensions sud-ouest de la typologie de Flouriot. Les grandes communes rurales périphériques et les communes à haut standing résidentiel (Gombe, Ngaliema, Limete) maintiennent des densités beaucoup plus faibles (inférieures à 20000 hab. $/ \mathrm{km}^{2}$ ) même si leur croissance est forte.

\section{Extension de la Ville de Kinshasa ou densification des quartiers existants?}

La croissance démographique entraîne une pression sur l'espace kinois car elle est à l'origine de l'extension de la zone bâtie en périphérie et de la densification des zones déjà bâties. Ces deux dernières sont en étroite relation via les migrations intraurbaines. En effet, la croissance de la population dans les communes périphériques est essentiellement due aux migrations issues d'autres communes de la ville, sans oublier le facteur de croissance naturelle, très important dans cette ville.

\section{Extension}

33 La comparaison entre les limites de la ville en 1969 et l'extension du bâti en 1995 permet de visualiser l'extension de la tache urbaine de Kinshasa (fig. 2). 

croissance observés entre les recensements de 1970 et de 1984 (figure 4).

Pour quantifier l'extension urbaine, on peut faire l'hypothèse que la proportion d'espace bâti en 1969 est identique à celle de 1995 pour les communes urbaines de 1969, c'est-à-dire que la croissance urbaine s'est réalisée uniquement par extension et non par densification. La tache urbaine est alors estimée à $147 \mathrm{~km}^{2}$ en 1969 et à $339 \mathrm{~km}^{2}$ en 1995, y compris les espaces interstitiels et espaces verts urbains. L'extension urbaine se serait effectuée à un rythme de $7,1 \mathrm{~km}^{2} /$ an sur ces 27 années. 1051000 d'habitants. ville en 1995, on estime la population de la ville en 1995 à 2423735 personnes. En 1995, d'après les projections de l'INS (1993), il y avait environ 4787000 habitants à Kinshasa. On peut donc affirmer que l'extension de Kinshasa a absorbé un peu plus du tiers de la croissance de la population alors que la densification des quartiers concerne un peu moins des deux tiers de cet accroissement.

Ces estimations relativisent le point de vue établi au départ de la carte des taux de Figure 4. Taux de croissance observés entre 1970 et 1984.

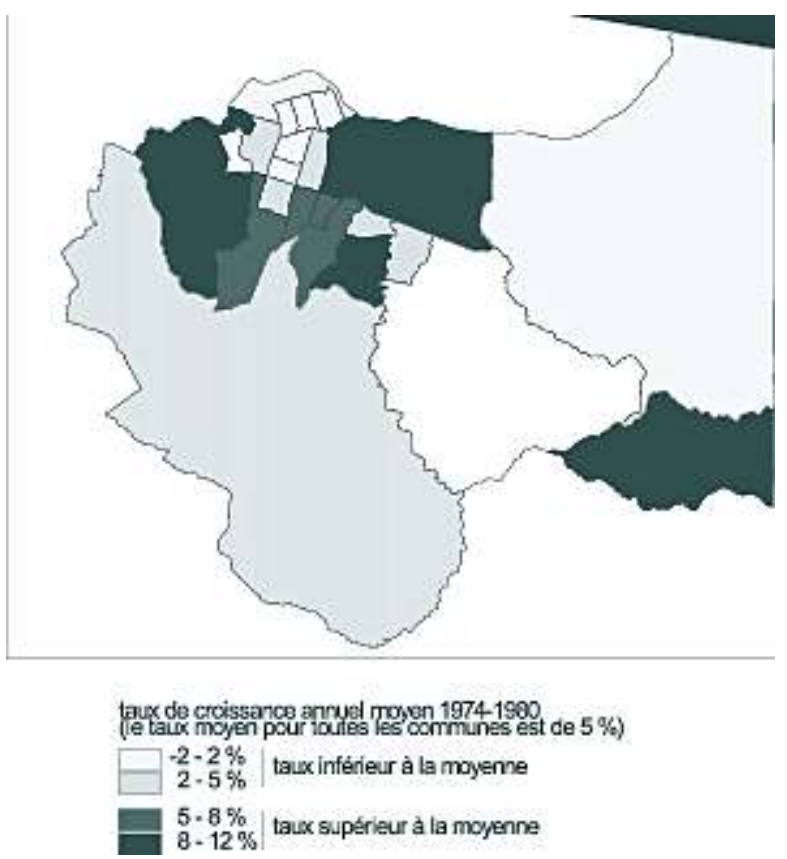

\section{Densification}

A Kinshasa, l'extrême concentration des fonctions et infrastructures, ainsi que les facilités relatives de transport et de raccordement aux réseaux de distribution d'eau et d'électricité, font des communes centrales des zones très attractives par rapport aux communes périphériques. Les terrains y sont en conséquence particulièrement chers. D'après nos enquêtes personnelles, en 2000, le prix de vente d'une parcelle à Kalamu 
s'élevait à 25000 US \$, alors qu'il était de 25 à 1250 US \$ à Mont Ngafula, et de 3000 US \$ à Masina. Les densités de population particulièrement élevées (de 20000 à 50000 hab./ $\mathrm{km}^{2}$ bâti) entraînent une grande promiscuité; en effet, d'après nos enquêtes personnelles, à Kalamu, 3 à 4 ménages vivent sur une même parcelle, alors qu'à Mont Ngafula ou à Kimbanseke, il n'y a qu'un ménage par parcelle.

La densification des communes centrales depuis 1984 est bien réelle mais elle semble avoir atteint ses limites, ce qui se traduit actuellement par des migrations du centreville vers des quartiers des communes intermédiaires ou périphériques, avec une localisation à proximité des axes de communication vers le centre-ville. Ce sont donc ces quartiers localisés à proximité des voies de communication qui se densifient.

Au Sud et à l'Ouest, on observe quelques zones d'habitat concentré dans les zones collinaires où l'habitat est majoritairement disséminé et entouré de végétation pour éviter les glissements de terrain. Ces zones d'habitat concentré se développent le long des routes principales. Elles résultent d'une densification de l'habitat: au sein de l'espace bâti, on divise les parcelles et on construit là où il reste de la place. Le but des nouveaux habitants est de s'éloigner au minimum du centre-ville tant en distance (localisation le plus près possible du centre-ville) qu'en temps de parcours (localisation à proximité des routes principales).

41 A l'Est, en dehors des communes rurales de Nsele et de Maluku, non seulement le bâti s'étend, mais il se densifie. L'urbanisation vers l'est de la ville, encouragée par les autorités locales, bénéficiait de la proximité d'axes routiers importants, de terrasses alluviales vastes et de terrains disponibles relativement peu coûteux. Aujourd'hui, les communes de Masina, Ndjili et Kimbanseke sont très peuplées, mais l'équipement et la voirie n'ont pas suivi l'urbanisation. En conséquence, l'accès aux voies de communication (localisées au nord de ces communes) au départ du front d'urbanisation (localisé au sud de ces communes) est de plus en plus difficile. La zone urbanisée ne peut donc s'étendre éternellement en l'absence de routes de liaison. C'est ainsi que l'on observe une extrême densification du bâti à proximité des axes routiers principaux, par exemple le long du Boulevard Lumumba et de la route de Mokali à Kimbanseke, mais aussi l'urbanisation d'espaces auparavant laissés "vierges", par exemple les zones inondables et insalubres de Masina.

\section{Conclusion}

L'utilisation d'une image satellitaire SPOT a permis de tracer les limites des communes de Kinshasa. Si le tracé des communes centrales était assez bien connu (mais non disponible sous forme numérique), celui des communes périphériques n'était pas cartographié dans les documents à notre disposition. Le calcul des superficies a mis en évidence des erreurs parfois non négligeables avec les superficies mentionnées dans le recensement de 1984.

Ensuite, une classification de l'image satellitaire SPOT a abouti à l'identification de l'espace bâti. Superposées aux limites communales dans un système d'information géographique, les superficies bâties par communes ont été comparées à la typologie des communes réalisée par Flouriot (1975). Ensuite, les densités de population ont été calculées par rapport à l'espace bâti. Enfin, ce calcul montre que l'extension de la ville 
de Kinshasa coexiste avec un phénomène de densification et que celui-ci absorbe près des deux tiers de l'accroissement de la population entre 1969 et 1995.

L'image satellitaire peut être avantageusement exploitée dans un contexte où les cartes urbaines sont complètement dépassées par le rythme de l'extension de la ville.

\section{BIBLIOGRAPHIE}

BRUNEAU J.-C. (1994), « Crise et déclin de la croissance des villes au Zaïre - Une image actualisée », Revue Belge de Géographie, $119^{\text {ème }}$ année, Bruxelles, Société Royale Belge de Géographie, pp. 103-114.

de MAXIMY R. (1984), Kinshasa, une ville en suspens..., ORSTOM, Paris.

de Saint Moulin L. (1976), Atlas des collectivités du Zaïre, Kinshasa, Presses Universitaires du Zaïre.

de SAINT MOULIN L. et LUTUTALA MUMPASI B. (1995), Réorientation des migrants vers les villes secondaires au Zaïre : évidences et contraintes des cas d'Inkisi et de Kikwit.

DUBRESSON A. et RAISON J.-P. (1998), L'Afrique subsaharienne - Une géographie du changement, Paris, Ed. Armand Colin.

FLOURIOT J., de MAXIMY R. et PAIN M. (1975), Atlas de Kinshasa, Toulouse, Université de Toulouse.

I.N.S. (1992), Totaux définitifs - groupements / quartiers. Volume 1 - Kinshasa, Bas-Zaïre, Bandundu, Equateur, Haut-Zaïre, Kinshasa, Institut National de la Statistique, Ministère du plan et aménagement du territoire, République du Zaïre.

I.N.S. (1993), Projections démographiques - Zaïre et Régions - 1984-2000, Kinshasa, Institut National de Statistique, Ministère du Plan et Aménagement du Territoire, République du Zaïre.

MWANZA Wa MWANZA H. (1996), Transport et implantation des équipements socio-collectifs dans une métropole tropicale: Kinshasa (Zaïre), Thèse présentée en vue de l'obtention du titre de doctorat en Sciences géographiques, Bruxelles.

NGONDO S., de SAINT MOULIN L., TAMBASHE B. (1992), Perspectives démographiques du Zaïre, 1984-1999 et population d'âge électoral en 1993 et 1994, Kinshasa, CEPAS (Centre d'Etudes Pour l'Action Sociale).

PAIN M., (1994), Kinshasa, la ville et la cité, ORSTOM, Collection Mémoires, n 105, Etudes Urbaines, Paris.

VENNETIER P. (1991), Les villes d'Afrique tropicale, Paris, éd. Masson - Géographie.

WOLFF E., MASHINI DHI MBITA MULENGHE J.-C., IPALAKA YOBWA J. et MASSART M. (2001), Organisation de l'espace et infrastructure urbaine en République Démocratique du Congo, Libreville, ADIE. 


\section{RÉSUMÉS}

L'utilisation d'images satellitaires SPOT de 1995 a permis de tracer les limites des communes de Kinshasa. Le calcul des superficies a mis en évidence des erreurs parfois non négligeables avec les superficies mentionnées dans le recensement de 1984. Ensuite, une classification de l'image satellitaire SPOT a abouti à l'identification de l'espace bâti. Superposées aux limites communales dans un système d'information géographique, les superficies bâties par commune ont été comparées à la typologie des communes réalisée par Flouriot (1975). Ensuite, les densités de population ont été calculées par rapport à l'espace bâti. Enfin, on montre que l'extension de la ville de Kinshasa coexiste avec un phénomène de densification et que la densification a absorbé près des deux tiers de l'accroissement de la population entre 1969 et 1995.

The use of SPOT images of 1995 allowed to trace the limits of the communes of Kinshasa. The measurement of their areas underlined significant errors compared to areas mentioned in the census of 1984. Then, a classification of the SPOT image led to the identification of built-up areas. Overlayed with the limits of the communes within a geographic information system, built-up areas per commune were compared to the typology of Flouriot (1975). Then, population densities were computed using the built-up area. Finally, we show that the extension of the city of Kinshasa co-exists with a densification phenomena and that densification absorbed nearly two third of the population increase between 1969 and 1995.

\section{INDEX}

Mots-clés : Kinshasa, République Démocratique du Congo, ville, densité de population, urbanisation, télédétection, bâti

Keywords : Kinshasa, Democratic Republic of Congo, city, population density, urbanisation, remote sensing, built-up

\section{AUTEURS}

\section{ELÉONORE WOLFF}

Laboratoire de géographie humaine, Université Libre de Bruxelles, ewolff@ulb.ac.be

\section{VIRGINIE DELBART}

Laboratoire de géographie humaine, Université Libre de Bruxelles 